\title{
Homogeneous Systems with a Quiescent Phase
}

\author{
K.P. Hadeler ${ }^{1}$ \\ Department of Mathematics and Statistics, Arizona State University, Tempe, AZ 85287, USA
}

\begin{abstract}
Recently the effect of a quiescent phase (or dormant/resting phase in applications) on the dynamics of a system of differential equations has been investigated, in particular with respect to stability properties of stationary points. It has been shown that there is a general phenomenon of stabilization against oscillations which can be cast in rigorous form. Here we investigate, for homogeneous systems, the effect of a quiescent phase, and more generally, a phase with slower dynamics. We show that each exponential solution of the original system produces two exponential solutions of the extended system whereby the stability properties can be controlled.
\end{abstract}

Key words: quiescence, homogeneous system, exponential solution, stability, non-linear eigenvalue problem, predator-prey system

AMS subject classification: 34C14, 34D08, 92D25, 92D40

\section{Introduction}

Vector fields of the same dimension can be diffusively coupled. Typically such coupling is mathematically interesting only if the vector fields are identical or somehow similar. In that case the coupled system is a very small version of a lattice dynamical system. An exception is the case when a given vector field is coupled to the zero vector field. This problem has many applications, in particular in biology, where the zero state can be interpreted as quiescence or dormancy [15], [10], [3], [13], [11], [8], [9], [2], [12], and, in a somewhat different context, [7]. For this reason we speak of systems with a quiescent phase. The fundamental mathematical question is easy to formulate. Suppose everything is known about the qualitative behavior of a dynamical system. What can be said about the qualitative behavior of the system extended by a quiescent phase which has twice the dimension? Does completeness imply completeness, does a global attractor result in

\footnotetext{
${ }^{1}$ E-mail: hadeler@uni-tuebingen.de
} 
a global attractor, what happens to isolated stationary points and periodic orbits? Such questions have been systematically investigated in two recent publications [6] and [5]. It has been shown, in quantitative terms, that introducing a quiescent phase stabilizes stationary points against the onset of Hopf bifurcations, i.e., for truly complex eigenvalues the real parts are decreased until they become negative. The effect is more pronounced if the imaginary parts are large. Eigenvalues on the imaginary axis other than 0 move into the left half-plane of the complex plane. For highly symmetric systems it has been shown that introducing a quiescent phase reduces the amplitude of an existing periodic orbit until the orbit shrinks to a point [5]. The results on stationary points make use of the fact that the stationary points of the given system and of the extended system are essentially the same. Treating periodic orbits in a general setting (without symmetry) is difficult since such correspondence does not exist for periodic orbits (at least we do not know).

As far as the mathematical theory is concerned, exponential solutions of homogeneous systems lie between stationary points and periodic orbits since periodic orbits are invariant under the group $S^{1}$ and exponential solutions are invariant under the group $\mathbb{R}_{+} \backslash\{0\}$. In both cases an individual solution can be fixed by a Poincaré map or transverse section. Therefore the stability problem should be easier for exponential solutions of homogeneous systems then for periodic orbits. This is in fact the case, as will be shown in the sequel. For basic properties of homogeneous systems we refer to [4]. In [4] there are also results on periodic solutions; we do not need them here.

One may ask whether the theory of quiescent phases can be extended by coupling the vector field $f$ to the vector field $\kappa f$, with $\kappa \in[0,1)$, instead to the zero field. We call such an extension, for $\kappa \in(0,1)$, a system with slower dynamics. It seems that in general such extension is not meaningful because one cannot even control the stationary points. However, for homogeneous vector fields the situation is different. We show for homogeneous vector fields with slower dynamics that to each exponential solution of the small system there are two exponential solutions of the extended system and further, that stability is preserved.

In section 2. we present basic facts on homogeneous systems. In section 3. we introduce systems with quiescent phase and we recall some essential properties. In section 4. we discuss homogeneous vector fields with quiescent phases and slower dynamics, in particular their exponential solutions. The main existence result states that for every exponential solution of the given system there are two exponential solutions of the system extended by slower dynamics. In section 5. we show a stability result: If an exponential solution of the given vector field is stable, then one of the corresponding exponential solutions of the extended field (the one with the larger exponent) is stable, the other is unstable.

In section 6. a two-dimensional predator-prey system is given as an example. The discussion of the four-dimensional problem with slower dynamics would be cumbersome while the general results yield existence and local stability easily.

\section{Homogeneous systems}

Let $F: \mathbb{R}^{m} \rightarrow \mathbb{R}^{m}$ be homogeneous of degree 1 , i.e.,

$$
F(\alpha x)=\alpha F(x) \text { for } \alpha>0 .
$$


Then

$$
\dot{x}=F(x)
$$

defines a homogeneous dynamical system. Suppose

$$
x(t)=\hat{x} e^{\hat{\lambda} t}
$$

is an exponential solution. Then the number $\hat{\lambda}$ and the vector $\hat{x}$ are an eigenvalue and eigenvector of the nonlinear eigenvalue problem

$$
F(\hat{x})=\hat{\lambda} \hat{x}
$$

and every solution to the nonlinear eigenvalue problem gives rise to an exponential solution. Notice that we consider only real eigenvalues. In the following we shall identify the pair $(\hat{\lambda}, \hat{x})$ with the exponential solution $\hat{x} \exp \{\hat{\lambda} t\}$. Of, course, except in the linear case $F(x)=A x$, the number of (nonlinear) eigenvectors need not be the same as the space dimension. In particular, it can be larger than the space dimension.

Now we introduce the appropriate notion of stability. This definition is analogous to the definition of orbital stability for periodic orbits. Suppose $(\hat{\lambda}, \hat{x})$ is an eigenvalue and eigenvector and (2.3) is the exponential solution. Let $\phi: \mathbb{R}^{m} \rightarrow \mathbb{R}^{m}$ be a smooth function which is homogeneous of degree 1 and $\phi(x)>0$ for $x \neq 0$. Then $\mathcal{S}=\left\{x \in \mathbb{R}^{m}: \phi(x)=1\right\}$ is a sphere and the projected system on the sphere is

$$
\dot{y}=F(y)-\phi^{\prime}(y) F(y) \cdot y .
$$

Exponential solutions of (2.2) correspond to stationary points of (2.5). An exponential solution is called stable (in the sense of homogeneous systems) if the corresponding stationary point $\hat{x}$ is stable, similarly for "linearly stable" and "asymptotically stable". The important point is that this notion of stability is independent of the "transverse section" $\phi$ and that linear stability can be checked in terms of the eigenvalues of the Jacobian matrix $A=F^{\prime}(\hat{x})$ rather then in terms of the Jacobian matrix of the projected system.

The Jacobian matrix $A$ is homogeneous of degree 0 in $\hat{x}$. From Euler's formula we have $A \hat{x}=F^{\prime}(\hat{x}) \hat{x}=\hat{\lambda} \hat{x}$. Hence $\hat{\lambda}$ is an eigenvalue of the linear operator $A: \mathbb{R}^{m} \rightarrow \mathbb{R}^{m}$ with eigenvector $\hat{x}$. The operator has, multiplicities counted, $m$ (complex in general) eigenvalues $\lambda_{1}=$ $\hat{\lambda}, \lambda_{2}, \ldots, \lambda_{m}$. The stability theorem for exponential solutions (see [4]) says that the exponential solution corresponding to the pair $(\hat{\lambda}, \hat{x})$ is linearly stable in the sense of homogeneous systems if and only if

$$
\Re \lambda_{k}<\hat{\lambda} \text { for } k=2, \ldots, m \text {. }
$$

\section{Quiescent phases}

Let $f: \mathbb{R}^{n} \rightarrow \mathbb{R}^{n}$ be a smooth vector field. Together with the dynamical system

$$
\dot{u}=f(u)
$$


we study the system in $\mathbb{R}^{2 n}$

$$
\begin{aligned}
\dot{v} & =f(v)-\gamma_{2} v+\gamma_{1} w \\
\dot{w} & =\gamma_{2} v-\gamma_{1} w
\end{aligned}
$$

with positive coupling constants $\gamma_{1}, \gamma_{2}$. Hence, in the system (3.2) the vector field $f$ is diffusively coupled to the zero vector field. Here the coupling rates are the same for all components. For the case of different rates (replace the matrices $\gamma_{i} I$ by positive diagonal matrices) see [6], [1].

Let $\bar{u}$ be a stationary point of (3.1), $f(\bar{u})=0$. Then $(\bar{v}, \bar{w})=\left(\bar{u},\left(\gamma_{2} / \gamma_{1}\right) \bar{u}\right)$ is a stationary point of (3.2). All stationary points of (3.2) are obtained in this way.

Let $A=f^{\prime}(\bar{u})$ be the Jacobian at $\bar{u}$. Then the Jacobian of $(3.2)$ at $(\bar{v}, \bar{w})$ is

$$
B=\left(\begin{array}{cc}
A-\gamma_{2} I & \gamma_{1} I \\
\gamma_{2} I & -\gamma_{1} I
\end{array}\right)
$$

If $\mu$ is an eigenvalue of $A$ then there are two corresponding eigenvalues $\lambda_{1}, \lambda_{2}$ of $B$, with $\Re \lambda_{2} \leq \Re \lambda_{1}$, which are solutions to the quadratic equation

$$
\lambda^{2}+\lambda\left(\gamma_{1}+\gamma_{2}-\mu\right)-\gamma_{1} \mu
$$

The properties of these eigenvalues in terms of $\mu$ are presented in the following theorem which has been proved in [6].

Theorem 1. The eigenvalues $\mu$ and $\lambda_{1}, \lambda_{2}$ are related as follows:

(a) Let $\mu=\alpha \in \mathbb{R}$. Then $\lambda_{1}, \lambda_{2}$ are real.

(a.i) If $\alpha<0$ then $\lambda_{2}<\alpha<\lambda_{1}<0$.

(a.ii) If $\alpha=0$ then $\lambda_{2}=-\left(\gamma_{1}+\gamma_{2}\right)<0=\lambda_{1}$.

(a.iii) If $\alpha>0$ then $\lambda_{2}<0<\lambda_{1}<\alpha$.

(b) Let $\mu=\alpha \pm i \beta, \beta>0$. Then $\Re \lambda_{2}<0$.

(b.i) If $\alpha \leq 0$ then $\Re \lambda_{1}<0$.

(b.ii) If $\alpha>0$ then $\Re \lambda_{1}<\alpha$.

(b.iii) If $\alpha \leq 0$ and $\beta^{2}+\left(\gamma_{1}+\gamma_{2}+\alpha\right)^{2}+4 \alpha \gamma_{2}>0$ and $\beta^{2}\left(\gamma_{1}+\alpha\right)+\alpha\left(\gamma_{1}+\gamma_{2}+\alpha\right)^{2}>0$, then $\Re \lambda_{1}<\alpha$.

(b.iv) If $\alpha>0$ and $\beta^{2}>4 \alpha \gamma_{1}-\left(\gamma_{1}+\gamma_{2}-\alpha\right)^{2}$ and $\beta\left(\gamma_{2}-\alpha\right)>\alpha\left(\gamma_{1}+\gamma_{2}-\alpha\right)^{2}$,

then $\Re \lambda_{1}<0$.

The theorem says that a quiescent phase damps high-frequency oscillations and stabilizes against Hopf bifurcations.

\section{Slowed dynamics}

If one thinks in chemical reaction terms, then the dynamics (3.1) describes a situation where a reaction takes place in the $v$ compartment while nothing happens in the $w$ compartment. One can 
also look at a situation with a slower reaction in the $w$ compartment. Then one has, instead of (3.2), the system

$$
\begin{aligned}
\dot{v} & =f(v)-\gamma_{2} v+\gamma_{1} w \\
\dot{w} & =\kappa f(w)+\gamma_{2} v-\gamma_{1} w
\end{aligned}
$$

with some $\kappa<1$. The case $\kappa=0$ corresponds to a quiescent phase. For $\kappa \neq 0$ there is, in general, no clear relation between the stationary points of (3.1) and those of the extended system (4.1). We shall see that the system (4.1) becomes more interesting when $f$ is assumed to be homogeneous.

Therefore we assume that the vector field $f$ in (3.1) is homogeneous of degree 1 . For $\kappa \in \mathbb{R}$ the extended vector field

$$
F\left(\begin{array}{c}
v \\
w
\end{array}\right)=\left(\begin{array}{c}
f(v)-\gamma_{2} v+\gamma_{1} w \\
\kappa f(w)+\gamma_{2} v-\gamma_{1} w
\end{array}\right)
$$

is also homogeneous of degree 1 and the results of section 2. apply.

We show that each exponential solution for $f$ produces two exponential solutions for $F$ whereby one of the two is always unstable. However, for $\kappa \neq 0$ there may be exponential solutions for $F$ which are not connected to exponential solutions for $f$. With respect to these additional solutions, we run into the same problem as described for (4.1) in the non-homogeneous case.

We include the case $\kappa=1$. We assume $\kappa \leq 1$ because the case $\kappa>1$ can be reduced to that case by exchanging the roles of $v$ and $w$. We call the system with $\kappa \in(0,1)$ a system with slower dynamics. We shall see that the cases $\kappa=0, \kappa \in(0,1)$, and $\kappa=1$ behave somewhat differently.

The following theorem is formally somewhat similar to Theorem 1 but Theorem 1 is about linear stability at stationary points while Theorem 2 is about existence of exponential solutions.

Theorem 2. i) Let $(\hat{\lambda}, \hat{v})$ be a nonlinear eigenvalue and eigenvector of $f$. Then there are two related nonlinear eigenvalues $\hat{\lambda}_{2} \leq \hat{\lambda}_{1}$ of $F$ with eigenvectors $\left(\hat{v}, \hat{w}_{1}\right)$ and $\left(\hat{v}, \hat{w}_{2}\right)$.

The eigenvalues are solutions to the equation

$$
\phi(\lambda) \equiv\left(\lambda-\hat{\lambda}+\gamma_{2}\right)\left(\lambda-\kappa \hat{\lambda}+\gamma_{1}\right)-\gamma_{1} \gamma_{2}=0 .
$$

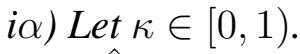

$$
\begin{aligned}
& \text { If } \hat{\lambda}>0 \text { then } \hat{\lambda}_{2}<\kappa \hat{\lambda}<\hat{\lambda}_{1}<\hat{\lambda} \text {. } \\
& \text { If } \hat{\lambda}=0 \text { then } \hat{\lambda}_{2}<0=\hat{\lambda}_{1}=\hat{\lambda} \text {. } \\
& \text { If } \hat{\lambda}<0 \text { then } \hat{\lambda}_{2}<\hat{\lambda}<\hat{\lambda}_{1}<\kappa \hat{\lambda} \text {. } \\
& \text { i } \beta) \text { Let } \kappa=1 \text {. Then } \hat{\lambda}_{1}=\hat{\lambda} \text { and } \hat{\lambda}_{2}=\hat{\lambda}-\left(\gamma_{1}+\gamma_{2}\right) \text {. } \\
& \text { ii) If } \kappa=0 \text { then all eigenvalues and eigenvectors are obtained in this way. }
\end{aligned}
$$

Proof:

i) Let $f(\hat{v})=\hat{\lambda} \hat{v}, \hat{v} \neq 0$. We look for an eigenvector of $F$ of the form $(v, w)=(\hat{v}, c \hat{v})$, with some $c \in \mathbb{R}$, with eigenvalue $\lambda$. Then we have necessarily

$$
\begin{aligned}
\hat{\lambda}-\gamma_{2}+\gamma_{1} c & =\lambda \\
c \kappa \hat{\lambda}+\gamma_{2}-\gamma_{1} c & =\lambda c .
\end{aligned}
$$


We eliminate $c$ and get equation (4.3). From (4.3) we we find

$$
\begin{aligned}
\phi(\hat{\lambda}) & =\gamma_{2}(1-\kappa) \hat{\lambda}, \\
\phi(\kappa \hat{\lambda}) & =-\gamma_{1}(1-\kappa) \hat{\lambda}
\end{aligned}
$$

from where the claims in i) are obvious.

ii) Let $\kappa=0$. We are looking for an eigenvector of $F$, i.e., for $(\lambda, v, w)$ satisfying

$$
\begin{aligned}
f(v)-\gamma_{2} v+\gamma_{1} w & =\lambda v \\
\gamma_{2} v-\gamma_{1} w & =\lambda w .
\end{aligned}
$$

Hence (unless $\lambda=-\gamma_{1}$ which leads to $v=w=0$ )

$$
f(v)=\left(\lambda+\gamma_{2}-\frac{\gamma_{1} \gamma_{2}}{\lambda+\gamma_{1}}\right) v .
$$

Hence the first component $v$ of the eigenvector is necessarily an eigenvector of $f$. We call that vector $v=\hat{v}$. Then

$$
\lambda+\gamma_{2}-\frac{\gamma_{1} \gamma_{2}}{\lambda+\gamma_{1}}=\hat{\lambda}
$$

and hence $\lambda$ is a solution of the quadratic equation (4.3). Finally

$$
w=\frac{\gamma_{2}}{\lambda+\gamma_{1}} .
$$

From Theorem 2 we know where $\hat{\lambda}_{1}$ is located with respect to $\hat{\lambda}$. For the following stability discussion we need more information on $\hat{\lambda}_{1}$. We consider $\hat{\lambda}_{1}$ as a function of $\hat{\lambda}$. The number $\hat{\lambda}_{1}$ is explicitly given as

$$
\begin{aligned}
2 \hat{\lambda}_{1} & =\left((1+\kappa) \hat{\lambda}-\left(\gamma_{1}+\gamma_{2}\right)\right) \\
& +\sqrt{(1-\kappa)^{2} \hat{\lambda}^{2}+2 \hat{\lambda}(1-\kappa)\left(\gamma_{1}-\gamma_{2}\right)+\left(\gamma_{1}+\gamma_{2}\right)^{2}} .
\end{aligned}
$$

Proposition 3. The number $\hat{\lambda}_{1}$ is an increasing function of $\hat{\lambda}$.

Proof: In view of $0 \leq \kappa \leq 1$ we have

$$
4\left(\gamma_{1}+\gamma_{2}\right)^{2}>\left(4-4 \kappa+\kappa^{3}\right)\left(\gamma_{1}-\gamma_{2}\right)^{2} .
$$

Working backwards, we find that this inequality is equivalent with

$$
\begin{gathered}
16 \kappa(1-\kappa)^{2}\left[\left(\gamma_{1}+\gamma_{2}\right)^{2}-(1-\kappa)^{2}\left(\gamma_{-} \gamma_{2}\right)^{2}\right] \\
>4\left(\gamma_{1}-\gamma_{2}\right)^{2}\left((1-\kappa)-(1-\kappa)^{3}\right)^{2} .
\end{gathered}
$$


The inequality (4.7) implies that the following quadratic in $\hat{\lambda} \in \mathbb{R}$ is always positive,

$$
\begin{gathered}
\hat{\lambda}^{2} 4 \kappa(1-\kappa)^{2}+2 \hat{\lambda}\left(\gamma_{-} \gamma_{2}\right)\left((1-\kappa)-(1-\kappa)^{3}\right) \\
+\left(\gamma_{1}+\gamma_{2}\right)^{2}-(1-\kappa)^{2}\left(\gamma_{1}-\gamma_{2}\right)^{2}>0 .
\end{gathered}
$$

The inequality (4.8) is equivalent with

$$
\begin{aligned}
& (1+\kappa)^{2}\left[(1-\kappa)^{2} \hat{\lambda}^{2}+\left(\gamma_{1}+\gamma_{2}\right)^{2}+2 \hat{\lambda}(1-\kappa)\left(\gamma_{1}-\gamma_{2}\right)\right] \\
& (1-\kappa)^{4} \hat{\lambda}^{2}+2 \hat{\lambda}(1-\kappa)^{3}\left(\gamma_{1}-\gamma_{2}\right)+(1-\kappa)^{2}\left(\gamma_{1}-\gamma_{2}\right)^{2} .
\end{aligned}
$$

By taking square roots on both sides we find

$$
\begin{gathered}
(1+\kappa) \sqrt{(1-\kappa)^{2} \hat{\lambda}^{2}+2 \hat{\lambda}(1-\kappa)\left(\gamma_{1}-\gamma_{2}\right)+\left(\gamma_{1}+\gamma_{2}\right)^{2}} \\
>-(1-\kappa)^{2} \hat{\lambda}-(1-\kappa)\left(\gamma_{1}-\gamma_{2}\right)
\end{gathered}
$$

or

$$
(1+\kappa)+\frac{(1-\kappa)^{2} \hat{\lambda}+(1-\kappa)\left(\gamma_{1}-\gamma_{2}\right)}{\sqrt{(1-\kappa)^{2} \hat{\lambda}^{2}+2 \hat{\lambda}(1-\kappa)\left(\gamma_{1}-\gamma_{2}\right)+\left(\gamma_{1}+\gamma_{2}\right)^{2}}}>0
$$

This inequality says, in view of (4.5), that

$$
2 \frac{d \hat{\lambda}_{1}}{d \hat{\lambda}}>0
$$

\section{Stability}

Now we discuss the stability problem. Even in the case $\kappa=0$ this problem cannot be reduced to section 3. and Theorem 1 because the projected system to a homogeneous system with quiescent phase is not a standard system with quiescent phase.

Let $(\hat{\lambda}, \hat{v})$ be an eigenvalue and eigenvector of $f$ and let $\left(\hat{\lambda}_{1}, \hat{v}, \hat{w}_{1}\right)$ and $\left(\hat{\lambda}, \hat{v}, \hat{w}_{2}\right)$ be the associated eigenvalues and eigenvectors of $F$. Let $A=f^{\prime}(\hat{v})$ be the Jacobian matrix of $f$. Then the Jacobian matrix of $F$ is given by

$$
B=\left(\begin{array}{cc}
A-\gamma_{2} I & \gamma_{1} I \\
\gamma_{2} I & \kappa A-\gamma_{1} I
\end{array}\right)
$$

and it is the same for both eigenvectors because these differ only in the component $w=c v$ and $f^{\prime}$ is homogeneous of degree 0 .

Assume that the matrix $A$ has the eigenvalues $\hat{\lambda}, \mu_{2}, \ldots, \mu_{n}$. The matrix $B$ has $2 n$ eigenvalues. To begin with, following section 4., there are the eigenvalues $\hat{\lambda}_{1}$ and $\hat{\lambda}_{2}$ which satisfy (4.3), and 
then, similar to the situation in Theorem 1, there are eigenvalues $\lambda_{k 1}$ and $\lambda_{k 2}$, with $\Re \lambda_{k 2} \leq \Re \lambda_{k 1}$ which satisfy

$$
\left(\lambda_{k i}-\mu_{k}+\gamma_{2}\right)\left(\lambda_{k i}-\kappa \mu_{k}+\gamma_{1}\right)-\gamma_{1} \gamma_{2}=0 .
$$

From the general framework in section 2. we obtain a first stability result. It says that, for a given exponential solution of the small system, of the two resulting exponential solutions of the large system only the one with the larger exponent may be stable, and the stability of the latter can be checked by inspecting $n$ eigenvalues rather than $2 n$.

Proposition 4. i) The exponential solution corresponding to $\left(\hat{\lambda}_{1}, \hat{v}, \hat{w}_{1}\right)$ is stable if

$$
\Re \lambda_{k 1}<\hat{\lambda}_{1}, \quad k=2, \ldots, n .
$$

ii) The exponential solution corresponding to $\left(\hat{\lambda}_{2}, \hat{v}, \hat{w}_{2}\right)$ is not stable.

Proof: i) According to section 2. we have to check whether all eigenvalues of $B$ except $\hat{\lambda}_{1}$ have real parts less than $\hat{\lambda}_{1}$. We know, by Theorem 1 and Proposition 2, that $\hat{\lambda}_{2}<\hat{\lambda}_{1}$ and $\Re \lambda_{k 2}<\Re \lambda_{k 1}$ for $k=2, \ldots, n$. Hence only the conditions (5.2) have to be checked.

ii) Since $\hat{\lambda}_{1}>\hat{\lambda}_{2}$, there is an eigenvalue of $B$ that is greater than $\hat{\lambda}_{1}$. Hence the solution for $\hat{\lambda}_{2}$ is not stable.

Now we can formulate the key stability result which says that stability is preserved in the transition from the small to the large system.

Theorem 5. Let $\kappa \in[0,1]$. Suppose the exponential solution corresponding to $(\hat{\lambda}, \hat{v})$ of $f$ is linearly stable in the sense of exponential solutions. Then the exponential solution corresponding to $\left(\hat{\lambda}_{1}, \hat{v}, \hat{w}_{1}\right)$ is linearly stable in the sense of exponential solutions.

The proof is performed in several steps. We have to show that

$$
\Re \mu_{k}<\hat{\lambda}, \quad k=2, \ldots, n
$$

implies

$$
\Re \lambda_{k 1}<\hat{\lambda}_{1}, \quad k=2, \ldots, n .
$$

The difficulty is that we should not compare the eigenvalues of the matrices $A$ and $B$ but those of $A-\hat{\lambda} I$ and $B-\hat{\lambda}_{1} I$. The proof is performed in two steps. By Proposition 3 we know that $\hat{\lambda}_{1}$ is an increasing function of $\hat{\lambda}$. This allows later to replace $\hat{\lambda}$ by the real part $\alpha$ of the eigenvalue under consideration. In the other step we compare real parts. We need the formula for the real part of a square root

$$
\Re \sqrt{\xi+i \eta}=\frac{1}{\sqrt{2}}\left[\xi+\sqrt{\xi^{2}+\eta^{2}}\right]^{1 / 2} .
$$

Let $\mu_{k}=\mu=\alpha+i \beta$ and $\lambda_{k 1}=\lambda_{1}$. The number $\lambda_{1}$ is given by

$$
\begin{aligned}
2 \lambda_{1}= & \left((1+\kappa) \mu-\left(\gamma_{1}+\gamma_{2}\right)\right) \\
& +\sqrt{(1-\kappa)^{2} \mu^{2}+2 \mu(1-\kappa)\left(\gamma_{1}-\gamma_{2}\right)+\left(\gamma_{1}+\gamma_{2}\right)^{2}}
\end{aligned}
$$


and hence its real part is given by

$$
\begin{aligned}
2 \Re \lambda_{1}= & (1+\kappa) \alpha-\left(\gamma_{1}+\gamma_{2}\right) \\
& +\frac{1}{\sqrt{2}}\left\{(1-\kappa)^{2}\left(\alpha^{2}-\beta^{2}\right)+2 \alpha(1-\kappa)\left(\gamma_{1}-\gamma_{2}\right)+\left(\gamma_{1}+\gamma_{2}\right)^{2}\right. \\
& +\left[\left[(1-\kappa)^{2}\left(\alpha^{2}-\beta^{2}\right)+2 \alpha(1-\kappa)\left(\gamma_{-} \gamma_{2}\right)+\left(\gamma_{1}-\gamma_{2}\right)^{2}\right]^{2}\right. \\
& \left.\left.+4 \beta^{2}(1-\kappa)^{2}\left[(1-\kappa) \alpha+\gamma_{1}-\gamma_{2}\right]^{2}\right]^{1 / 2}\right\}^{1 / 2} .
\end{aligned}
$$

Define a number $\alpha_{1}$ by

$$
\begin{aligned}
2 \alpha_{1}= & (1+\kappa) \alpha-\left(\gamma_{1}+\gamma_{2}\right) \\
& +\sqrt{(1-\kappa)^{2} \alpha^{2}+2 \alpha(1-\kappa)\left(\gamma_{1}-\gamma_{2}\right)+\left(\gamma_{1}+\gamma_{2}\right)^{2}} .
\end{aligned}
$$

Proposition 6.

$$
\Re \lambda_{1}<\alpha_{1} .
$$

Proof: We start from $\left(\gamma_{1}-\gamma_{2}\right)^{2}<\left(\gamma_{1}+\gamma_{2}\right)^{2}$, multiply both sides by $4 \beta^{2}(1-\kappa)^{2}$ and rearrange terms. Then we get the inequality

$$
\begin{gathered}
{\left[(1-\kappa)^{2}\left(\alpha^{2}-\beta^{2}\right)+2 \alpha(1-\kappa)\left(\gamma_{1}-\gamma_{2}\right)+\left(\gamma_{1}+\gamma_{2}\right)^{2}\right]^{2}} \\
+4 \beta^{2}(1-\kappa)^{2}\left((1-\kappa) \alpha+\gamma_{1}-\gamma_{2}\right]^{2} \\
<\left[(1-\kappa)^{2}\left(\alpha^{2}+\beta^{2}\right)+2 \alpha(1-\kappa)\left(\gamma_{1}-\gamma_{2}\right)+\left(\gamma_{1}+\gamma_{2}\right)^{2}\right]^{2} .
\end{gathered}
$$

We take square roots on both sides,

$$
\begin{aligned}
& \left\{\left[(1-\kappa)^{2}\left(\alpha^{2}-\beta^{2}\right)+2 \alpha(1-\kappa)\left(\gamma_{1}-\gamma_{2}\right)+\left(\gamma_{1}+\gamma_{2}\right)^{2}\right]^{2}\right. \\
& \left.+4 \beta^{2}(1-\kappa)^{2}\left((1-\kappa) \alpha+\gamma_{1}-\gamma_{2}\right]^{2}\right\}^{1 / 2} \\
& <(1-\kappa)^{2}\left(\alpha^{2}+\beta^{2}\right)+2 \alpha(1-\kappa)\left(\gamma_{1}-\gamma_{2}\right)+\left(\gamma_{1}+\gamma_{2}\right)^{2}
\end{aligned}
$$

and rearrange again

$$
\begin{aligned}
& (1-\kappa)^{2}\left(\alpha^{2}-\beta^{2}\right)+2 \alpha(1-\kappa)\left(\gamma_{1}-\gamma_{2}\right)+\left(\gamma_{1}+\gamma_{2}\right)^{2} \\
& \quad+\left\{\left[(1-\kappa)^{2}\left(\alpha^{2}-\beta^{2}\right)+2 \alpha(1-\kappa)\left(\gamma_{1}-\gamma_{2}\right)+\left(\gamma_{1}+\gamma_{2}\right)^{2}\right]^{2}\right. \\
& \left.\quad+4 \beta^{2}(1-\kappa)^{2}\left((1-\kappa) \alpha+\gamma_{1}-\gamma_{2}\right]^{2}\right\}^{1 / 2} \\
& <2\left[(1-\kappa)^{2} \alpha^{2}+2 \alpha(1-\kappa)\left(\gamma_{1}-\gamma_{2}\right)+\left(\gamma_{1}+\gamma_{2}\right)^{2}\right] .
\end{aligned}
$$

Now we divide by 2 , take square roots on both sides and add $(1+\kappa) \alpha-\left(\gamma_{1}+\gamma_{2}\right)$ on both sides. Then we arrive at the desired inequality.

Proof of Theorem 5: We have shown in Proposition 6 that $\Re \lambda_{1}<\alpha_{1}$. We have shown in Proposition 3 that $\hat{\lambda}_{1}$ is an increasing function of $\hat{\lambda}$. By assumption $\alpha<\hat{\lambda}$. Hence $\alpha_{1}<\hat{\lambda}_{1}$ and hence $\Re \lambda_{1}<\hat{\lambda}_{1}$. 


\section{Example}

As an example we choose a homogeneous predator-prey system

$$
\begin{aligned}
\dot{u} & =a u-b \frac{u v}{u+v} \\
\dot{v} & =c \frac{u v}{u+v}-d v
\end{aligned}
$$

with $a, b, c, d>0$. If this system is projected to the unit interval $[0,1]$ parameterized by $\xi=$ $x /(x+y)$ then we get the the scalar equation

$$
\dot{\xi}=\xi(1-\xi)[a+d-b-(c-b) \xi]
$$

and exponential solutions become stationary points. From the scalar equation (6.2) we can determine the stationary points and their stability and hence the existence of exponential solutions and their stability. However, we cannot determine the exponents from (6.2).

The example behaves somewhat similar to a Lotka competition model or the Fisher-WrightHaldane model for two alleles. There are always the pure states $\xi=0$ and $\xi=1$. The behavior is determined by inequalities between $b, c$, and $a+d$.

I) $c<a+d<b$ : There is a coexistence point, it is unstable and separates the basins of attraction of 0 and 1 .

II) $a+d<b$ and $a+d<c$ : There is no coexistence point. The point 0 attracts $[0,1)$.

III) $c>a+d>b$ : There is a coexistence point. It is globally attracting in $(0,1)$.

IV) $a+d>b$ and $a+d>c$ : There is no coexistence point. The point 1 attracts $(0,1]$.

In cases I) and III) the exponent of the coexistence solution is

$$
\rho=\frac{b c-a c-b d}{b-c} .
$$

The exponent $\rho$ is negative in the unstable case I and positive in the stable case III.

While these observations are easy to verify, the behavior of the four-dimensional problem with slower dynamics becomes quite cumbersome. Projecting does not help because the projected system has dimension 3. However, the results of the previous sections tell that to each stable exponential solution corresponds one stable exponential solution. In particular, in case III, the system with slowed dynamics has a stable exponential solution where prey and predator coexist.

\section{References}

[1] L. Bilinsky, K.P. Hadeler. Quiescence stabilizes predator-prey relations. Submitted.

[2] G. Carrero, D. McDonald, E. Crawford, G. de Vries, M. Hendzel. Using FRAP and mathematical modeling to determine the in vivo kinetics of nuclear proteins. Methods, 29 (2003), $14-28$. 
[3] M. Gyllenberg, G.F. Webb. Quiescence as an explanation of Gompertzian tumor growth. Growth, Development, and Aging, 53 (1989), 25-33.

[4] K.P. Hadeler. Periodic solutions of homogeneous equations. J. Diff. Equ. 95 (1992), 183202.

[5] K.P. Hadeler, T. Hillen. Coupled dynamics and quiescent phases. In: Math Everywhere Deterministic and Stochastic Modelling in Biomedicine, Economy and Industry. (Proc. Conf. Milano 2005), 7-23, Springer Verlag, Berlin, 2007.

[6] K.P. Hadeler. Quiescent phases and stability. Lin. Alg. Appl. 428 (2008), 1620-1627.

[7] K.P. Hadeler. The role of migration and contact distribution in epidemic spread. In C. Castillo-Chavez and H.T. Banks, editors, Frontiers Appl. Math., 28, Bioterrorism, 188210, SIAM, 2003.

[8] K.P. Hadeler, M.A. Lewis. Spatial dynamics of the diffusive logistic equation with a sedentary compartment. Canadian Appl. Math. Quart., 10 (2002), 473-499.

[9] T. Hillen. Transport equations with resting phases. Europ. J. Appl. Math., 14 (2003), 613636.

[10] W. Jäger, S. Krömker, B. Tang. Quiescence and transient growth dynamics in chemostat models. Math. Biosci., 119 (1994), 225-239.

[11] M.A. Lewis, G. Schmitz, Biological invasion of an organism with separate mobile and stationary states: modeling and analysis. Forma, 11 (1996), 1-25.

[12] T. Malik, H.L. Smith. A resource-based model of microbial quiescence. J. Math. Biol., 53 (2006), 231-252.

[13] M.G. Neubert, P. Klepac, P. van den Driessche. Stabilizing dispersal delays in predator-prey metapopulation models. Theor. Popul. Biol., 61 (2002), 339-347.

[14] E. Pachepsky, F. Lutscher, R.M. Nisbet, M. Lewis. Persistence, spread and the drift paradox. Theor. Pop. Biol., 67 (2005), 61-73.

[15] B. Tang. Mathematical models of microbial competition in laboratory cultures incorporating environmental heterogeneities: coexistence. Surveys Math. Indust., 3 (1993), 49-70. 